\title{
Molecular Characterization and Cross-Reactivity of Feline Calicivirus Circulating in Southwestern China
}

\author{
Long Zhou ${ }^{1,2,+}{ }^{+}$, Nengsheng Fu ${ }^{1,+}$, Lu Ding ${ }^{1}$, Yan $\mathrm{Li}^{1,2}{ }^{1}$, Jian Huang ${ }^{1,2}$, Xue Sha ${ }^{1}$, Qun Zhou ${ }^{1}$, Xin Song ${ }^{1}$ \\ and Bin Zhang 1,2,*(D) \\ 1 College of Animal \& Veterinary Sciences, Southwest Minzu University, Chengdu 610041, China; \\ zhoulongscu@163.com (L.Z.); fls17865690921@sina.com (N.F.); Ningning20002021@163.com (L.D.); \\ ajhans@126.com (Y.L.); huangjian.1122@163.com (J.H.); ssx311@163.com (X.S.); qunyushan@yeah.net (Q.Z.); \\ songxin116@sina.com (X.S.) \\ 2 Key Laboratory of Ministry of Education and Sichuan Province for Qinghai-Tibetan Plateau Animal Genetic \\ Resource Reservation and Utilization, Southwest Minzu University, Chengdu 610041, China \\ * Correspondence: binovy@sina.com; Tel.: +86-28-8552-8276 \\ + These authors contributed equally to this work.
}

Citation: Zhou, L.; Fu, N.; Ding, L.; Li, Y.; Huang, J.; Sha, X.; Zhou, Q.; Song, X.; Zhang, B. Molecular Characterization and Cross-Reactivity of Feline Calicivirus Circulating in Southwestern China. Viruses 2021, 13, 1812. https://doi.org/10.3390/ v13091812

Academic Editor: Chao-Nan Lin

Received: 19 August 2021

Accepted: 10 September 2021

Published: 12 September 2021

Publisher's Note: MDPI stays neutral with regard to jurisdictional claims in published maps and institutional affiliations.

Copyright: (C) 2021 by the authors. Licensee MDPI, Basel, Switzerland. This article is an open access article distributed under the terms and conditions of the Creative Commons Attribution (CC BY) license (https:// creativecommons.org/licenses/by/ $4.0 /)$.

\begin{abstract}
Feline calicivirus (FCV) is an important pathogen of cats that has two genogroups (GI and GII). To investigate the prevalence and molecular characteristics of FCVs in southwestern China, 162 nasal swab samples were collected from cats in animal shelters and pet hospitals. In total, 38 of the clinical samples (23.46\%) were identified as FCV positive using nested RT-PCR. Phylogenetic analyses using 10 capsid protein VP1 sequences revealed that 8 GI and 2 GII strains formed two independent clusters. Additionally, three separated FCVs that were not clustered phylogenetically (two GI and one GII strains) were successfully isolated from clinical samples and their full-length genomes were obtained. Phylogenetic and recombinant analyses of a GI FCV revealed genomic breakpoints in ORF1 and ORF2 regions with evidence for recombinant events between GI sub-genogroups, which is reported in China for the first time. Furthermore, sera obtained from mice immunized independently with the three FCV isolates and a commercial vaccine were used to evaluate the cross-reactivity of neutralizing antibodies. The three separate FCVs were neutralized by each other at a 1:19 to 1:775 titer range, whereas the triple-inactivated vaccine was at a titer of 1:16, which suggested that different genogroup/sub-genogroup FCV strains exhibit significantly different titers of neutralizing antibodies, including the commercial FCV vaccine. Thus, our study revealed the genetic diversity and complex cross-reactivity levels of FCVs in southwestern China, which provides new insights for application in vaccination strategies.
\end{abstract}

Keywords: feline calicivirus; molecular characterization; phylogenetic analysis; cross-reactivity; neutralizing antibodies

\section{Introduction}

Feline calicivirus (FCV), which belongs to the family Caliciviridae, genus Vesivirus, is the causative agent of respiratory disease, stomatitis, arthritis, and hemorrhagic-like fever in cats [1]. The virus was first isolated in 1957 [2] and is now widely distributed in many European, American, and Asian counties [3-5]. Generally, an FCV infection causes apparent or relatively mild oral cavity and upper respiratory tract symptoms $[6,7]$. Occasionally, FCV causes ulcerative dermatitis, severe pneumonia, feline chronic gingivostomatitis complex, abortion, acute jaundice, and acute febrile lameness syndrome [8,9]. However, in the past decade, virulent mutants of FCV called VS-FCVs, have been identified as the causal agents of severe and acute virulent systemic disease (VSD), resulting in a high persistent fever and high mortality rate in vaccinated cats [10,11].

The FCV genome is a non-enveloped, single-stranded positive-sense RNA of approximately $7.7 \mathrm{~kb}$ having three open reading frames (ORFs), including ORF1, ORF2, and 
ORF3 [12]. The ORF2 (2013 nt) encodes the capsid protein VP1 and is divided into six regions $(\mathrm{A}-\mathrm{F})$. The regions $\mathrm{A}, \mathrm{B}, \mathrm{D}$, and $\mathrm{F}$ are relatively well conserved, whereas regions $\mathrm{C}$ and $\mathrm{E}$ are variable with significant sequence divergence levels [13]. Therefore, the VP1 gene is frequently used as a target in molecular epidemiological investigations $[4,14,15]$. In accordance with the genetic diversity of the VP1 gene, the global FCVs are classified into two genogroups: genogroup I (GI) and genogroup II (GII) [14]. In China, most FCV strains belong to GII, and they are closely related to Japanese FCV strains $[5,16]$. It is worthy to note that new FCV strains have been constantly emerging in recent years owing to the large degree of viral genetic variation [17-19].

Vaccination against FCV has been the mainstay control method for decades [20-22]. Although several commercial vaccines are widely used in the field and effectively reduce the clinical disease incidence, it is not clear whether the existing commercial vaccines have cross-protection against the newly emerging FCV strains. In the present study, 162 suspected FCV clinical samples were collected from nasal swabs from cats in southwest China from 2017 to 2020, and then the molecular characterization, virus isolation, and cross-reactivity of FCV strains were further investigated.

\section{Materials and Methods}

\subsection{Sample Information and Treatment}

A total of 162 nasal swab samples were collected from cats suspected to be infected with FCV owing to symptomology that included conjunctivitis, respiratory issues (such as coughing, sneezing), and nasal discharge. The cats were being treated in 2 pet hospitals and 14 animal shelters in the Chengdu and Chongqing Regions, southwestern China, from 2017 to 2020. The cats in the pet hospitals were life-long household animals. The shelters received cats from different places, and they were grouped in cages for a short period of time with veterinary care. The mean geographical distance among the collection sites was about $500 \mathrm{~km}$ including the two cities. The ages of the cats ranged from 2 months to 3 years old, and the weight ranged from 0.6 to $8.9 \mathrm{~kg}$. All cats in this study were vaccinated with tripleinactivated feline Rhinotracheitis-Calici-Panleukopenia vaccines from Zoetis (ParsippanyTroy Hills, NJ, USA) or Boehringer Ingelheim (Ingelheim, Germany) $(1 \mathrm{~mL} /$ dose) by intramuscular injection. According to the instructions of the vaccines, the two companies use the same strains, including feline rhinotracheitis virus (FRV-605 strain), feline calicivirus (FCV-255 strain), and feline panleukopenia virus (FPV-Cu-4 strain) as the triple-inactivated vaccine for production. Each sample was homogenized separately in phosphate-buffered saline (PBS) and centrifuged at 10,000 r/min for $10 \mathrm{~min}$. The centrifugal supernatants were maintained at $-80^{\circ} \mathrm{C}$ until use.

\subsection{Primer Design}

One pair of nested RT-PCR primers was used to detect FCV from clinical samples as previously described [23], and another pair of primers (FCV-VP1-F/FCV-VP1-R) was designed for amplifying the VP1 gene hypervariable (VP1 HV) region of FCV (Table 1). The complete genomic sequences of three FCV stains were amplified with six overlapping fragments (Table S1) based on the full-length FCV reference sequences downloaded from NCBI GenBank.

\subsection{RNA Extraction and RT-PCR for VP1 Gene}

Viral RNA was extracted from sample homogenate with RNAiso Plus (TaKaRa, Dalian, China) according to the manufacturer's instructions. The cDNA was reverse transcribed using a PrimeScript ${ }^{\mathrm{TM}} \mathrm{RT}$ kit (TaKaRa, Dalian, China) following the manufacturer's instructions. The VP1 HV gene of FCV strains in this study were amplified using the VP1 primer pair (Table S1), and the PCR products were purified and cloned into pMD19-T vector (TaKaRa, Dalian, China). Then, the cloned viral cDNAs were sequenced by the Sanger sequencing approach (Sangon Biotech, Shanghai, China). 
Table 1. Information on the clinical specimens in this study.

\begin{tabular}{|c|c|c|c|c|c|c|c|}
\hline No. & Collection Site & Year & $\begin{array}{c}\text { No. of } \\
\text { Samples }\end{array}$ & $\begin{array}{c}\text { No. of } \\
\text { Positives }\end{array}$ & Positive Rate (\%) & Location & Clinical Sign \\
\hline 1 & Chengdu & 2017 & 5 & 5 & 100 & Shelter & $\begin{array}{c}\text { Conjunctivitis; nasal } \\
\text { discharge }\end{array}$ \\
\hline 2 & Chengdu & 2017 & 5 & 0 & 0 & Shelter & Nasal discharge \\
\hline 3 & Chongqing & 2018 & 8 & 4 & 50 & Shelter & Conjunctivitis \\
\hline 4 & Chengdu & 2018 & 15 & 6 & 40 & Shelter & Conjunctivitis \\
\hline 5 & Chongqing & 2018 & 11 & 3 & 27.3 & Shelter & Conjunctivitis \\
\hline 6 & Chengdu & 2018 & 4 & 2 & 50 & Shelter & Nasal discharge \\
\hline 7 & Chongqing & 2018 & 6 & 2 & 33.3 & Shelter & Nasal discharge \\
\hline 8 & Chengdu & 2018 & 5 & 1 & 20 & Shelter & Nasal discharge \\
\hline 9 & Chengdu & 2018 & 6 & 2 & 33.3 & Shelter & Nasal discharge \\
\hline 10 & Chengdu & 2018 & 4 & 2 & 20 & Shelter & Nasal discharge \\
\hline 11 & Chongqing & 2018 & 3 & 2 & 66.7 & Shelter & Sneeze; nasal discharge \\
\hline 12 & Chengdu & 2019 & 3 & 1 & 33.3 & Shelter & Nasal discharge \\
\hline 13 & Chengdu & 2019 & 5 & 0 & 0 & Shelter & Sneeze; conjunctivitis \\
\hline 14 & Chongqing & 2019 & 4 & 0 & 0 & Shelter & Sneeze; conjunctivitis \\
\hline 15 & Chongqing & 2020 & 20 & 2 & 10 & $\begin{array}{c}\text { Pet } \\
\text { Hospital }\end{array}$ & $\begin{array}{c}\text { Conjunctivitis; nasal } \\
\text { discharge }\end{array}$ \\
\hline 16 & Chengdu & 2020 & 58 & 6 & 10.3 & $\begin{array}{c}\text { Pet } \\
\text { Hospital }\end{array}$ & $\begin{array}{c}\text { Conjunctivitis; nasal } \\
\text { discharge }\end{array}$ \\
\hline Total & - & - & 162 & 38 & 23.46 & - & - \\
\hline
\end{tabular}

\subsection{Virus Isolation and Complete Genome Amplification}

The suspensions were passed through $0.22 \mu \mathrm{m}$ filters and inoculated into the Crandell Reese Feline Kidney (CRFK) cell monolayer and cultured in DMEM supplemented with $5 \%$ FBS (HyClone, Logan, UT, USA) at $37{ }^{\circ} \mathrm{C}$ in a $5 \% \mathrm{CO}_{2}$ incubator. Cell cultures were monitored every day for a cytopathic effect (CPE). If CPE appeared, the cells were freezethawed three times to release the virus. The viruses were further purified by plaque assay as previously described [16], and the purified viral isolates were cultured in monolayers of CRFK cells. The TCID 50 of the FCV isolates were calculated using the method of Muench and Reed [24]. The supernatants of the CPE-positive cultures were negatively stained using $0.5 \%$ phosphotungstic acid (Thermo Fisher, Waltham, MA, USA) and observed by electron microscopy. The complete genomes of the FCV isolates were further amplified using the primers above (Table S1). The sequences of six overlapping fragments from FCV isolates were assembled into full-length genome sequences using the SeqMan program in Lasergene software (DNASTAR, Madison, WI, USA).

\subsection{FCV Sequences Analysis}

Multiple sequence comparisons at nucleotide and amino acid levels were performed by the MegAlign program in Lasergene software7.2 (DNASTAR, Madison, WI, USA). Phylogenetic analysis of the VP1 HV gene and full-length FCV genomic sequences was performed using MEGA7.1 software (http:/ / www.megasoftware.net, access time: 21 January 2021) (Tempe, AZ, USA) with the distance-based neighbor-joining method. The bootstrap values were calculated on 1000 replicates. All reference sequences used to construct phylogenetic trees in this study were downloaded from NCBI GenBank. To identify potential recombination events, the complete genomic sequences of FCV isolates were compared with the reference strains. The aligned nucleotide sequences of the complete genomes were analyzed using the SimPlot software (version 3.5.1) (JHK University, Baltimore, MD, USA) with a $500 \mathrm{bp}$ window and a $20 \mathrm{bp}$ step. In addition, phylogenetic trees based on each recombinant fragment were constructed to confirm the accuracy of recombination events. 


\subsection{Preparation of Antigen and Serum Virus Neutralization Assay for FCV Isolates}

FCV s were propagated in CRFK cells and were inactivated with $1.0 \% \beta$-propiolactone (Thermo Fisher, Waltham, MA, USA) for $8 \mathrm{~h}$. Fifteen 6-week-old BALB/c female mice were randomly divided into five groups. Each group with 3 mice were immunized subcutaneously with condensed FCV-inactivated cell cultures mixed with Freund's complete adjuvant and the commercial triple-inactivated vaccine (Zoetis), respectively, followed by two booster immunizations. The mice in the negative control group were inoculated with cell culture medium with Freund's complete adjuvant. The serum virus neutralization assays were performed as follows: the sera were diluted 1:2, 1:4, 1:8, 1:16, 1:32, 1:64, 1:128, 1:256, 1:512, 1:1024, and 1:2048 by DMEM. The diluted sera were mixed with equal volumes of different FCV isolates $\left(200 \mathrm{TCID}_{50}\right)$ or commercial vaccine and incubated at $37^{\circ} \mathrm{C}$ for $1 \mathrm{~h}$. Then, the antibody-virus mixture was inoculated into CRFK monolayer cells, and the neutralizing antibody titers were obtained based on the estimation of $50 \%$ virus infection in cells as end points.

\section{Results}

\subsection{RT-PCR Survey of Clinical Samples}

In this study, 38 out of the 162 clinical samples were identified as positive for FCV using nested RT-PCR amplification, resulting in a positivity rate of $23.46 \%(38 / 162,95 \%$ CI: 17.2-30.7\%). Among them, 30 samples tested positive from 84 cats in 14 animal shelters (positive rate: $35.71 \%, 95 \%$ CI: $25.6-46.9 \%$ ), and 8 samples tested positive from 78 cats in 2 pet hospitals (positive rate: $10.26 \%, 95 \%$ CI: $4.5-19.2 \%$ ) (Table 1). Thus, the cats in the animal shelters had a significantly higher positive rate than those in the pet hospitals $(p<0.05)$.

\subsection{Sequence Alignment and Phylogenetic Analysis of the VP1 Gene}

Of the 38 positive samples, $10 \mathrm{VP} 1$ hypervariable regions (HVs) were successfully obtained by sequencing and designated as SMU-B5, SMU-Y5, SMU-B9, SMU-B22, SMUF4, SMU-Y14, SMU-C17, SMU-20, SMU-R1, and SMU-R55 (GenBank no.: MW194992MW195001). Sequence alignment showed that the nucleotide similarity of the 10 VP1 HVs ranged from $72.5 \%$ to $92.8 \%$, with $80.9-96.8 \%$ similarity levels in the deduced amino acid sequences. In addition, these viruses shared 72.0-89.2\% nucleotide homology and 77.8-92.4\% amino acid homology with the 35 reference FCV sequences from GenBank.

Phylogenetic analysis showed that the FCV strains clustered into two genogroups, GI and GII, as previously described [14], and the GI group is further classified into five clusters (I-V). Eight strains (SMU-B5, SMU-Y5, SMU-B9, SMU-Y14, SMU-C20, SMU-C17, SMU$\mathrm{R} 1$, and SMU-F4) were divided into three clusters in the GI group. Among these strains, SMU-B5, SMU-Y5, and SMU-B9 are closely related to strain FCV-21223 from the USA, and SMU-Y14 and SMU-C20 share close genetic relationships with the strains HRB-SS and XH from China. Additionally, three strains (SMU-R1, SMU-F4, and SMU-C17) were classified into a minor branch together with the Chinese strains GX2019 and GX01-13 from Guangxi province. The remaining two strains (SMU-R55 and SMU-B22) belong to the GII group and are closely related to strain ym3/2001/JP from Japan (Figure 1). No strains in this study were divided into cluster II of the GI group, which contains the representative vaccine strains FCV-255 (KM111170), FCV-2024 (AF479590), and F9 (M86379), suggesting that the FCV strains identified in vaccinated cats certainly cannot be related to used vaccines. Thus, FCV strains present in southwestern China are genetically diverse and complex.

\subsection{Isolation and Identification of FCV Strains}

Four separated strains (SMU-B22, SMU-F4, SMU-Y14, and SMU-B5) that did not cluster phylogenetically were filtered out for further viral isolation, and three strains (designated SMU-B22-2020, SMU-F4-2020, and SMU-B5-2020) were successfully isolated from the clinical samples using CRFK cells. The infected CRFK cells exhibited cytogamy, cell rounding, karyopyknosis, and cluster-like forms; they eventually detached completely 
and remained suspended (Figure 2a, SMU-B22-2020 isolate shown only). No obvious $\mathrm{CPE}$ was observed in the control cells (Figure $2 \mathrm{~b}$ ). The three isolates were subjected to electron microscope observation after three rounds of plaque purification. Spherical viral particles with no envelopes were observed, and these features are consistent with the structural characteristics of caliciviruses (Figure 2c). The viruses were further conformed by RT-PCR using the FCV specific primers (Figure 2d). The TCID 50 values of FCV SMUB22-2020, SMU-F4-2020, and SMU-B5-2020 were determined as $1 \times 10^{9.5} / 0.1,1 \times 10^{11} / 0.1$, and $1 \times 10^{8.5} / 0.1 \mathrm{~mL}$, respectively.

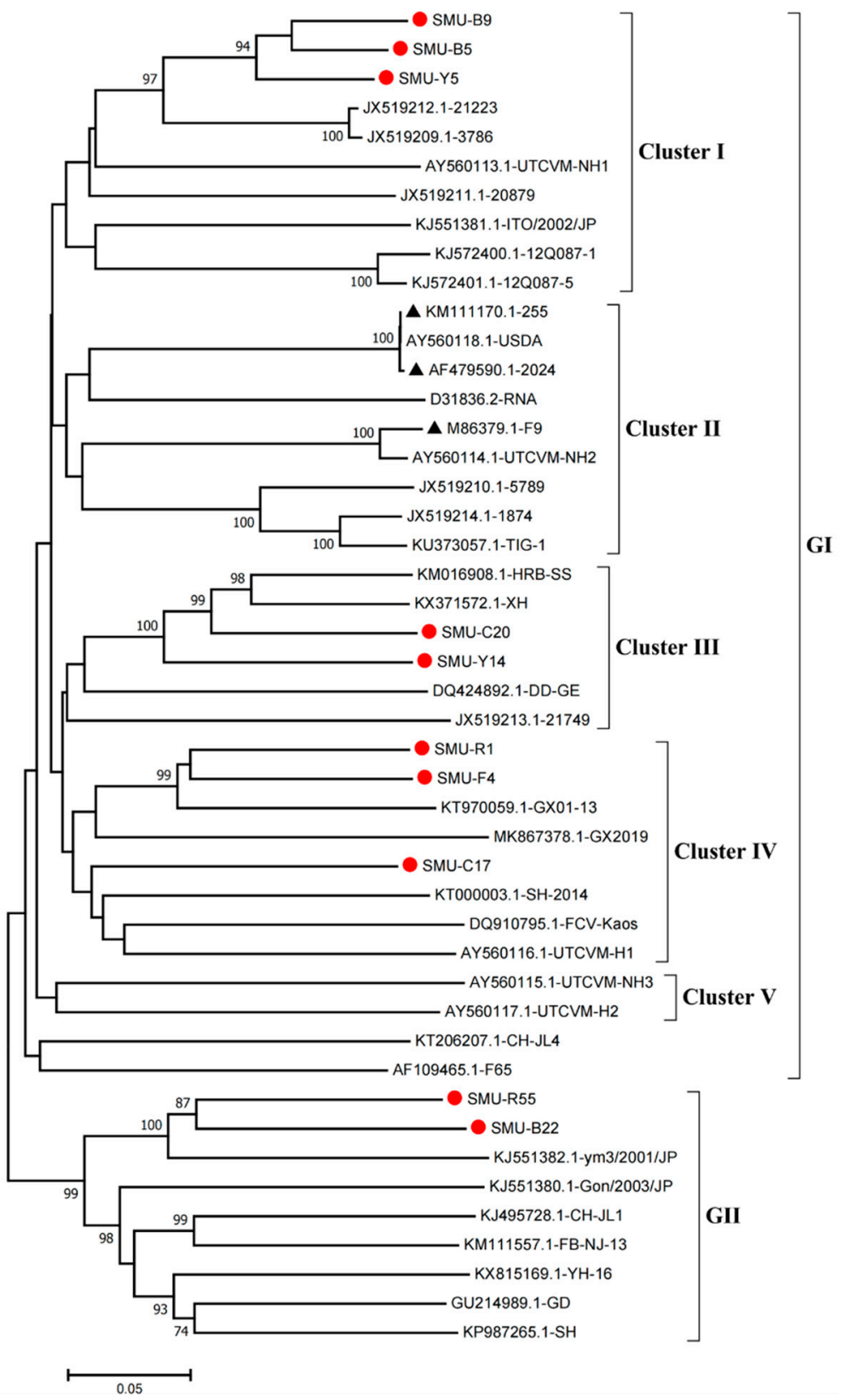

Figure 1. Phylogenetic tree of FCVs from southwestern China and 35 reference strains based on nucleotide sequences of VP1 HV. The phylogenetic tree was constructed with MEGA7.1 software (Tempe, AZ, USA) by the neighbor-joining method with 1000 bootstrap values. The viral assembly sequences characterized in this study are marked by "red circles" and the vaccine strains are labeled with "black triangles". The scale bar indicates the number of nucleotide substitutions per site. 

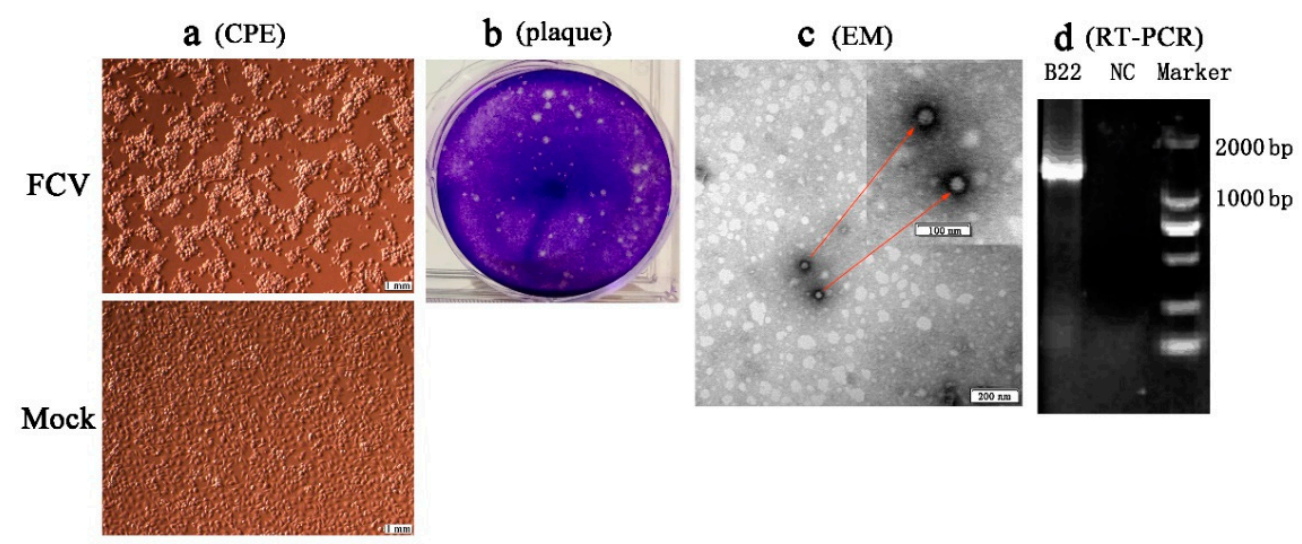

Figure 2. Isolation and identification of an FCV strain (SMU-B22-2020) in this study. (a) CRFK cells were inoculated with FCV SMU-B22-2020 and uninfected medium is shown at $12 \mathrm{~h}$ postinfection; (b) plaque assay for SMU-B22-2020 isolate with a dilution of $10^{-10}$ of virus stock using CRFK cells; (c) FCV particles with a diameter of approximately $30 \mathrm{~nm}$ were observed in cell supernatants by transmission electron microscopy (EM). Red arrows indicate the FCV particles; (d) the FCV isolate was further confirmed by RT-PCR with a $1300 \mathrm{bp}$ target fragment. NC indicates the negative control.

\subsection{Full-Length Genome Sequence Analyses of Three New FCV Isolates}

To further understand the genetic variation of the FCV strains in southwestern China, the full-length or nearly full-length genomes of three novel strains were successfully amplified from the positive FCV samples. The results have been submitted to the GenBank database (accession no.: MW194989-MW194991), and showed 7653 bp, 7686 bp, and $7705 \mathrm{bp}$ in length, respectively. The genetic analyses using MegAlign software revealed that the nucleotide identity ranged from $76.8 \%$ to $80.9 \%$, and the sequences shared $76.1-82.9 \%$ nucleotide identity levels with 31 other full-length genome reference sequences.

To examine the genomic characteristics of the three FCV strains, the nucleotide (nt) and amino acid (aa) homology levels of the FCV ORFs among the three strains in this study and with the 31 representative strains were compared (Figure 3a,b). For SMU-B22-2020, ORF1 and ORF3 shared 83.7-86.6\% nt (93.5-94.5\% aa) homology levels with CH-JL1, which was the highest homology level among the reference strains, whereas ORF2 of SMU-B22-2020 shared a $78.7 \%$ nt homology with $\mathrm{YH}-16$ and an $89.2 \%$ aa homology with $\mathrm{SH}$, which was a higher percentage than with other reference strains. For SMU-F4-2020, ORF1-3 shared $85.0 \% / 95.5 \%, 83.5 \% / 91.4 \%$, and 86.3\%/97.2\% nt/aa homology levels with GX2019, GX0113 , and 12Q087-5, respectively, which were higher than the homology levels with other reference strains. For SMU-B5-2020, ORF1-3 shared their highest nt/aa homology levels with HRB-SS (81.9\%/93.2\%), FCV-21223 (83.2\%/91.8\%), and UTCVM-NH3 (91.3\%/97.2\%), respectively. Thus, recombination events among different viruses might have occurred in their genomes.

Furthermore, we constructed phylogenetic trees using the complete genomes to determine the genetic relationships of the three isolates. The complete genome of the SMU-B52020 strain belonged to cluster III of the GI group and shared a close genetic relationship with two USA strains, UTCVM-NH1 and 20879. The SMU-F4-2020 strain clustered in cluster IV of the GI group and shared close relationships with two Chinese strains, CH-JL4 (northeastern China) and GX2019 (southern China). In addition, the SMU-B22-2020 strain was the neighbor of YH-16 and CH-JL1 in the GII group (Figure 4). The results suggest that various FCV strains from different clusters are simultaneously circulating and spreading in southwestern China. 

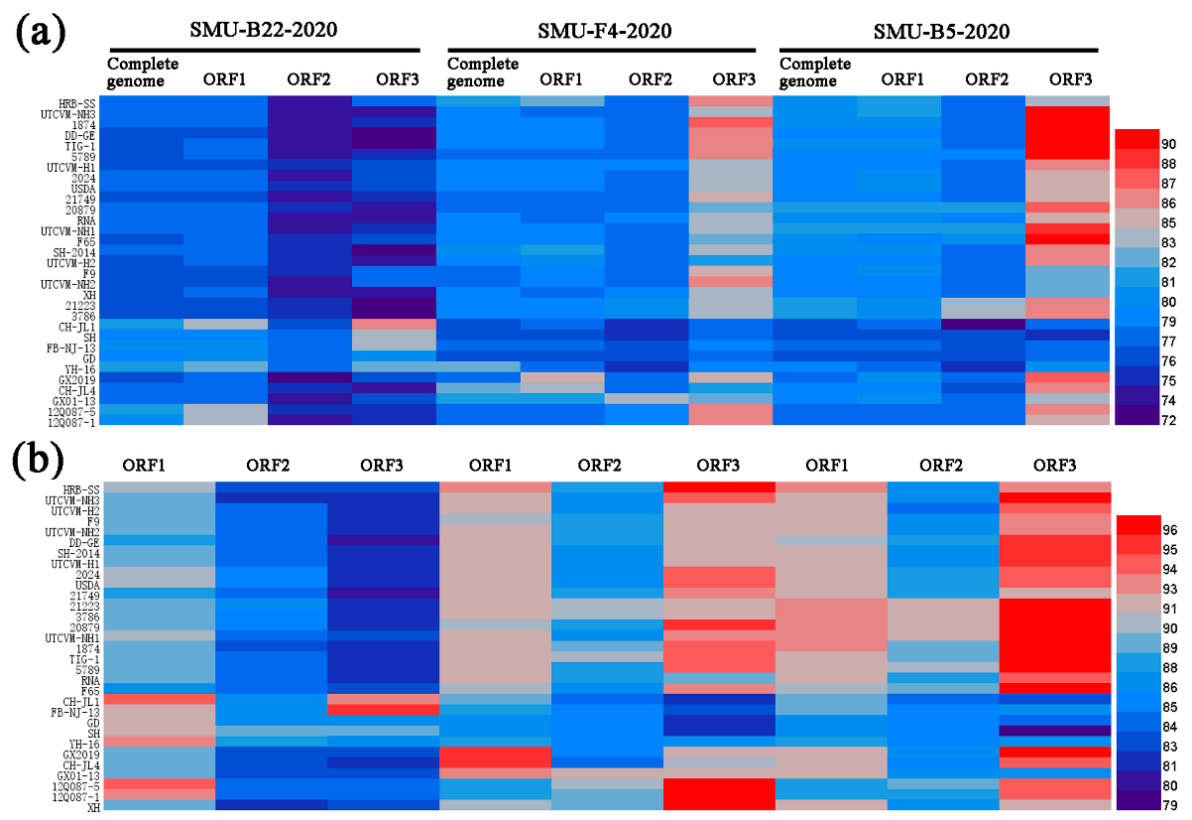

Figure 3. A heatmap was constructed based on the nucleotide sequence (a) and amino acid identities (b) of each genome among different FCV strains using HemI software. The colors represent sequence identity (blue for lower identities and red for higher identities) among different strains. The bar on the right represents the hierarchical similarity among the 15 meta-clusters (metric, Euclidean distance; linkage, average).

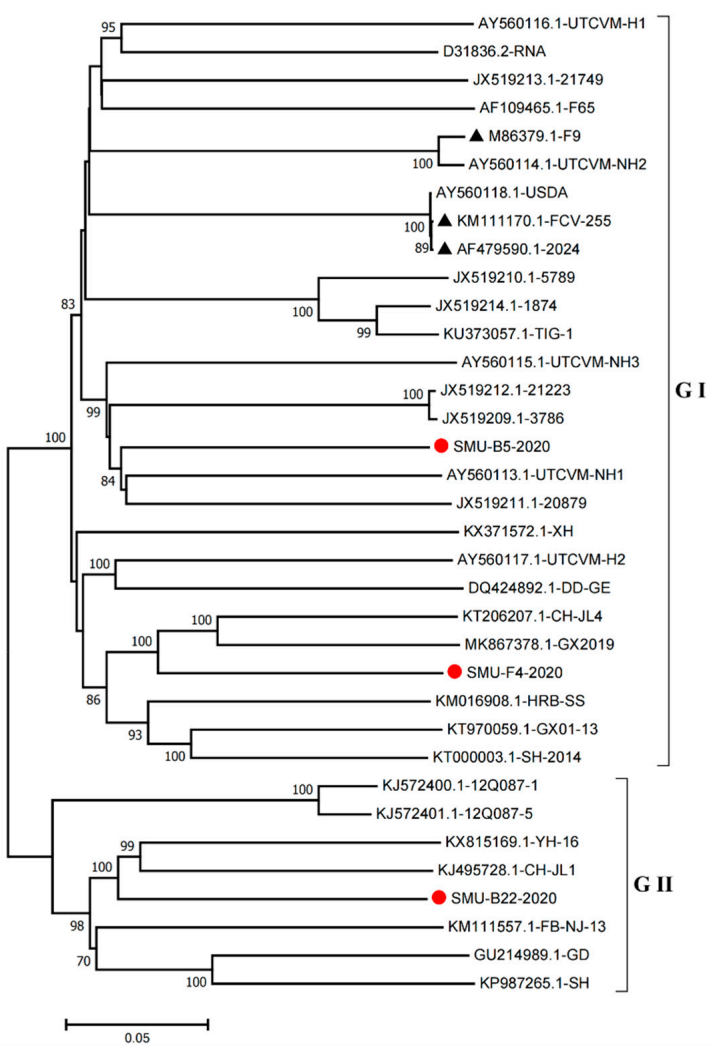

Figure 4. Phylogenetic tree based on full-length genomic sequence of three isolates (SMU-B22-2020, SMU-F4-2020, and SMU-B5-2020) and FCV reference strains available in GenBank. The three isolates in this study are labeled with "red circles" and the vaccine strains are labeled with "black triangles". The scale bar indicates the number of nucleotide substitutions per site. 
To identify possible recombinant events, we detected recombination using SimPlot and RDP4 software packages. The SMU-B5-2020 strain showed potential recombinant events between the FCV-21223 strain (cluster I of GI) and the HRB-SS strain (cluster III of GI) with $p$-values of $<1 \times 10^{-6}$ from the results of five detection methods. From the similarity plot, two recombination breakpoints within the SMU-B5-2020 genome were identified in ORF1 (nt 3104) and ORF2 (nt 5328) (Figure 5a). The breakpoints in SMU-B5-2020 separated its genome into three regions, with regions A (nt 1-3104) and C (nt 5328-7705) being closely related to the FCV-21223 strain, whereas region B (nt 3104-5328) was closely related to the HRB-SS strain (Figure 5b). The results indicated that the SMU-B5-2020 strain likely originated from recombination events between members of clusters I and III of the GI FCV strains.

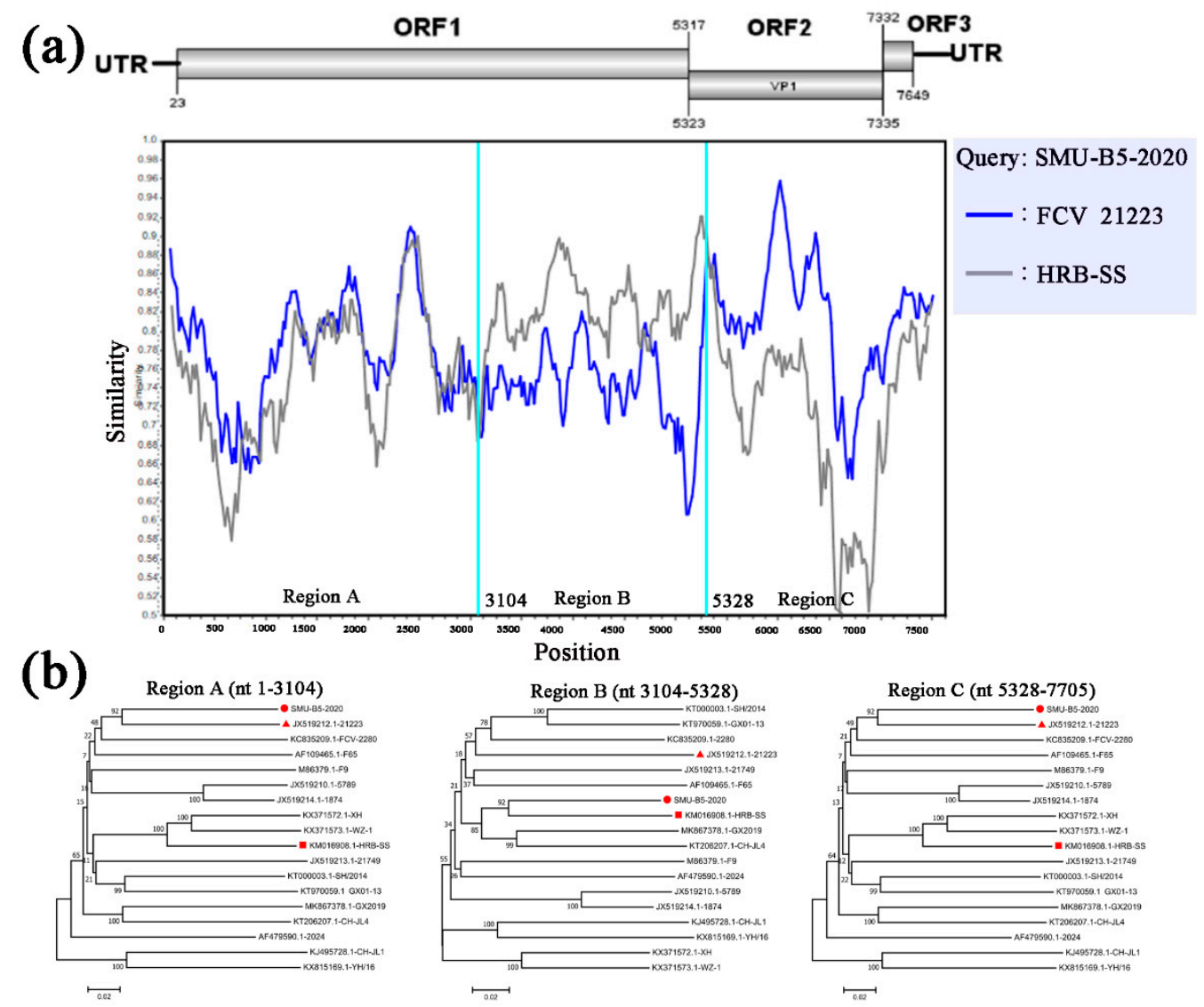

Figure 5. Full-length genome recombination analysis of SMU-B5-2020. (a) Genome scale similarity comparisons of SMU-B5-2020 (query) with FCV-21223 (blue) and HRB-SS (gray). The supposed recombination region is shown with red colors and the recombination breakpoints are marked at the bottom with nucleotide sites and viral genome structure referenced to FCV-21223; (b) phylogenetic trees based on every recombinant fragment within SMU-B5-2020 and 17 reference FCV strains are shown below the similarity plot. The strain SMU-B5-2020 is labeled with "red circles" and the putative recombinant parent strains are marked with "red triangles".

\subsection{Serum Neutralization Antibody Titers}

Viral antigen was prepared by mixing inactivated FCVs with Freund's complete adjuvant. A serum virus neutralization assay showed that the SMU-F4-2020-neutralizing antibody titer level in three mice reached as high as 1:775 \pm 65 to the SMU-F4-2020 isolate, which was significantly higher than the titers to SMU-B22-2020, SMU-B5-2020, and the vaccine $(p<0.05)$. Similarly, the SMU-B22-2020-neutralizing antibody titer level reached 1:655 \pm 55 to the SMU-B22-2020 strain, which was significantly higher than the titers to the other two isolates and the commercial vaccine $(p<0.05)$. The SMU-B5-2020-neutralizing 
antibody titer was 1:416 \pm 7.5 to SMU-B5-2020, which was significantly higher than the titers to the other two isolates and the commercial vaccine $(p<0.05)$ (Table 2$)$. The results indicated that the FCV-antibodies against each isolate did not provide better cross-reactivity between heterologous FCV isolates.

Table 2. Determination of serum neutralizing antibody titers to FCV isolates in mice.

\begin{tabular}{cccc}
\hline \multirow{2}{*}{ Neutralizing Antibody (NA) } & \multicolumn{3}{c}{ FCV Isolates } \\
\cline { 2 - 4 } & SMU-F4-2020 & SMU-B22-2020 & SMU-B5-2020 \\
\hline SMU-F4-2020-NA & $1: 775 \pm 65^{\mathrm{a}}$ & $1: 21.5 \pm 2.5$ & $1: 19 \pm 0$ \\
SMU-B22-2020-NA & $1: 106.6 \pm 0.5$ & $1: 655 \pm 55$ & $1: 63.5 \pm 0.5$ \\
SMU-B5-2020-NA & $1: 95 \pm 5$ & $1: 54.5 \pm 9.5$ & $1: 416.5 \pm 7.5$ \\
Vaccine-NA & $1: 16 \pm 0$ & $1: 16 \pm 0$ & $1: 16 \pm 0$ \\
\hline
\end{tabular}

a The neutralizing antibody titer is shown as mean \pm standard deviation (SD).

\section{Discussion}

In the general cat population, FCV infections are widespread and can cause respiratory disease, stomatitis, arthritis, and hemorrhagic-like fever [1]. At present, several types of vaccines are available for FCV. Although these commercial vaccines have been widely used in the field, FCV disease still remains a problem in cats. Vaccination against FCV has effectively reduced the clinical disease incidence but has not prevented highly virulent FCV strain (such as VS-FCV associated with VSD) outbreaks from occurring in vaccinated cats $[6,9,25]$. The frequent mutations of field FCV strains results in the poor protective effects of these vaccines. In this study, molecular epidemiological investigations and serum virus neutralization antibody assays of FCV in southwestern China were performed.

The prevalence of FCV is usually proportional to the number of cats in a household, with a higher infection rate usually occurring when large groups of cats are housed together. Therefore, pet cats kept in homes at low densities generally have a relatively low infection rate (approximately 10\%) [26]. In contrast, cats living in animal shelters with large populations usually have a higher chance of being infected (approximately $25-40 \%$ ) [3,27-29]. In China, the majority of FCV strains have been detected in domestic and stray cats $[16,30]$. To date, FCV strains from diseased cats were mainly reported in central and northeastern China $[5,16,31,32]$, with limited molecular epidemiological information from southwestern China. In this study, 162 nasal swab samples collected from cats in southwestern China (Chengdu and Chongqing) were subjected to FCV detection. The total positivity rate was $23.46 \%$, with cats in animal shelters having a significantly higher positive rate $(35.71 \%)$ than those in households $(10.26 \%)$, which is consistent with previous studies $[3,7,27]$. The result indicated that the infection rate is indeed associated with the number of cats.

VP1 is the most variable FCV structural protein and is composed of six regions (A-F). The variable region E contains major B-cell epitopes, which are targets of virusneutralizing antibodies [33]. Thus, VP1 is often used as a target for clinical diagnoses and new vaccine development. Phylogenetic analysis using the VP1 gene showed that FCV strains originating from southwestern China displayed abundant genetic diversity. All the detected FCV strains in this study clustered into two genogroups (GI and GII), and 8 out of 10 strains clustered into five clusters of GI, and the remaining 2 strains belonged to GII. Thus, the predominant viruses in southwest China were GI strains, which differs to a previous report in central China [5]. Full-length genome sequence analysis of three new FCV isolates (SMU-B22, SMU-F4, SMU-Y14, and SMU-B5) revealed that they shared 76.1-82.9\% nucleotide identity levels with reference strains and had the HVs in the VP1 gene. Phylogenetic analysis indicated that the three isolates were divided into different groups (two GI and one GII strains). Notably, the three FCV isolates in this study showed a distant genetic relationship with the vaccine strains FCV-2024 (AF479590) and F9 (M86379) based on whole-genome sequences, which suggested that the vaccines may not provide effective crossing protection against the epidemic FCV strains. 
Recombination is pervasive among RNA viruses and is a major mechanism contributing to the emergence and evolution of new variant strains $[34,35]$. Recombination was observed in an FCV strain W112 from the UK [36] and a 14Q315 strain from the Republic of Korea [19]. In the present study, the SMU-B5-2020 strain showed evidence of recombination events between cluster I and III members of GI FCV strains, which is reported in China for the first time. Considering its parental strains are FCV 21223 from the USA and HRB-SS from Heilongjiang (northeastern China), we speculate that the SMU-B5-2020 strain may have undergone a complicated evolutionary process.

Mutation is another important mechanism of FCV evolution that leads to the genetic divergence of FCVs in the field [1]. However, it is unclear whether mutations of the FCVs will affect the immuno-protection provided by commercial vaccines. A recent study showed that antibodies raised to the FCV-F9 vaccine remain broadly cross-reactive to separate FCV field strains from six European countries as determined by an in vitro neutralization assay [21]. In this study, to evaluate the cross-reactivity between different FCV isolates in China, a serum virus neutralization assay was performed. The FCV-antibody provided greater antibody neutralization reactivity against homologous strains (titer 1:416.5 to 1:775) than heterologous strains (titer 1:19 to 1:106). In addition, the vaccine-neutralizing antibodies titer was 1:16 to FCV strains, which was lower than the neutralizing antibodies raised by FCV isolates. Additionally, due to the samples in this study being collected from vaccinated cats with obvious clinical symptoms, we speculate that the current commercial vaccine does not appear to provide enough cross-protection to the contemporary FCV epidemic strains in this study. Furthermore, the immuno-protection of current commercial vaccines needs to be further confirmed by animal experiments.

\section{Conclusions}

In summary, the present study describes the epidemiological characteristics of FCV strains isolated in southwestern China during 2017-2020, and the predominant strains were the GI type. One FCV isolate was found to originate from recombination events among different GI strains. Further serum virus neutralization assays suggested that FCV antisera had significantly different cross-reactivity levels to temporally separated FCV populations. Our study highlights the importance of continuously monitoring FCV strains and provides new insights into the application of vaccination strategies in China.

Supplementary Materials: The following are available online at https://www.mdpi.com/article/ 10.3390/v13091812/s1, Table S1: Primers used to amplify the VP1 HV and full-length genome of FCV strains in this study.

Author Contributions: Conceptualization, L.Z. and N.F.; methodology, L.D.; software, L.Z.; formal analysis, Y.L. and J.H.; investigation, N.F. and X.S. (Xue Sha); resources, J.H., Q.Z., and X.S. (Xin Song); writing—original draft preparation, L.Z. and N.F.; writing—review and editing, L.Z. and B.Z; funding acquisition, B.Z. All authors have read and agreed to the published version of the manuscript.

Funding: This research was funded by the Applied Basic Research Project of Sichuan Province, China (grant no. 2020YJ0247), the National Natural Science Foundation of China (grant no.31772766), and the Southwest Minzu University Talent Supporting Funds (grant no. RQD2021004).

Institutional Review Board Statement: The study was approved by the Animal Care and Use Committee of Southwest Minzu University, Chengdu, Sichuan. The sampling was approved by the Animal Disease Control Center of Sichuan province, China (protocol code SYXK2011-043). All animals were released unharmed after sampling.

Informed Consent Statement: Not applicable.

Data Availability Statement: All sequences cloned in this study have been submitted to GenBank (GenBank accession no.: MW194992-MW195001 and MW194989-MW194991).

Acknowledgments: This study was supported by the College of Animal \& Veterinary Sciences, Southwest Minzu University, and Key laboratory of Ministry of Education and Sichuan Province 
for Qinghai-Tibetan Plateau Animal Genetic Resource Reservation and Utilization, Southwest Minzu University.

Conflicts of Interest: The authors declare no conflict of interest.

\section{References}

1. Radford, A.D.; Coyne, K.P.; Dawson, S.; Porter, C.J.; Gaskell, R.M. Feline calicivirus. Vet. Res. 2007, 38, 319-335. [CrossRef]

2. Fastier, L.B. A new feline virus isolated in tissue culture. Am. J. Vet. Res. 1957, 18, 382-389.

3. Bannasch, M.J.; Foley, J.E. Epidemiologic evaluation of multiple respiratory pathogens in cats in animal shelters. J. Feline Med. Surg. 2005, 7, 109-119. [CrossRef] [PubMed]

4. Hou, J.; Sanchez-Vizcaino, F.; McGahie, D.; Lesbros, C.; Almeras, T.; Howarth, D.; O'Hara, V.; Dawson, S.; Radford, A.D. European molecular epidemiology and strain diversity of feline calicivirus. Vet. Rec. 2016, 178, 114-115. [CrossRef] [PubMed]

5. Sun, Y.; Deng, M.; Peng, Z.; Hu, R.; Chen, H.; Wu, B. Genetic and phylogenetic analysis of feline calicivirus isolates in china. Vet. J. 2017, 220, 24-27. [CrossRef]

6. Pedersen, N.C.; Elliott, J.B.; Glasgow, A.; Poland, A.; Keel, K. An isolated epizootic of hemorrhagic-like fever in cats caused by a novel and highly virulent strain of feline calicivirus. Vet. Microbiol. 2000, 73, 281-300. [CrossRef]

7. Radford, A.D.; Sommerville, L.M.; Dawson, S.; Kerins, A.M.; Ryvar, R.; Gaskell, R.M. Molecular analysis of isolates of feline calicivirus from a population of cats in a rescue shelter. Vet. Rec. 2001, 149, 477-481. [CrossRef]

8. Schorr-Evans, E.M.; Poland, A.; Johnson, W.E.; Pedersen, N.C. An epizootic of highly virulent feline calicivirus disease in a hospital setting in New England. J. Feline Med. Surg. 2003, 5, 217-226. [CrossRef]

9. Hurley, K.E.; Pesavento, P.A.; Pedersen, N.C.; Poland, A.M.; Wilson, E.; Foley, J.E. An outbreak of virulent systemic feline calicivirus disease. J. Am. Vet. Med. Assoc. 2004, 224, 241-249. [CrossRef]

10. Reynolds, B.S.; Poulet, H.; Pingret, J.L.; Jas, D.; Brunet, S.; Lemeter, C.; Etievant, M.; Boucraut-Baralon, C. A nosocomial outbreak of feline calicivirus associated virulent systemic disease in france. J. Feline Med. Surg. 2009, 11, 633-644. [CrossRef]

11. Battilani, M.; Vaccari, F.; Carelle, M.S.; Morandi, F.; Benazzi, C.; Kipar, A.; Dondi, F.; Scagliarini, A. Virulent feline calicivirus disease in a shelter in italy: A case description. Res. Vet. Sci. 2013, 95, 283-290. [CrossRef] [PubMed]

12. Pesavento, P.A.; Chang, K.O.; Parker, J.S. Molecular virology of feline calicivirus. Vet. Clin. N. Am. Small Anim. Pract. 2008, 38, 775-786. [CrossRef] [PubMed]

13. Zang, M.; He, W.; Du, F.; Wu, G.; Wu, B.; Zhou, Z. Analysis of the codon usage of the orf2 gene of feline calicivirus. Infect. Genet. Evol. 2017, 54, 54-59. [CrossRef]

14. Sato, Y.; Ohe, K.; Murakami, M.; Fukuyama, M.; Furuhata, K.; Kishikawa, S.; Suzuki, Y.; Kiuchi, A.; Hara, M.; Ishikawa, Y.; et al Phylogenetic analysis of field isolates of feline calcivirus ( $\mathrm{fcv}$ ) in japan by sequencing part of its capsid gene. Vet. Res. Commun. 2002, 26, 205-219. [CrossRef] [PubMed]

15. Henzel, A.; Sa e Silva, M.; Luo, S.; Lovato, L.T.; Weiblen, R. Genetic and phylogenetic analyses of capsid protein gene in feline calicivirus isolates from rio grande do sul in southern brazil. Virus Res. 2012, 163, 667-671. [CrossRef] [PubMed]

16. Zhao, Y.; Chen, X.; Ying, Y.; Wang, K.; Dong, H.; Gao, C.; Yang, S.; Hu, G. Isolation and phylogenetic analysis of three feline calicivirus strains from domestic cats in jilin province, china. Arch. Virol. 2017, 162, 2579-2589. [CrossRef] [PubMed]

17. Caringella, F.; Elia, G.; Decaro, N.; Martella, V.; Lanave, G.; Varello, K.; Catella, C.; Diakoudi, G.; Carelli, G.; Colaianni, M.L.; et al Feline calicivirus infection in cats with virulent systemic disease, italy. Res. Vet. Sci. 2019, 124, 46-51. [CrossRef] [PubMed]

18. Di Martino, B.; Lanave, G.; Di Profio, F.; Melegari, I.; Marsilio, F.; Camero, M.; Catella, C.; Capozza, P.; Banyai, K.; Barrs, V.R.; et al. Identification of feline calicivirus in cats with enteritis. Transbound. Emerg. Dis. 2020, 67, 2579-2588. [CrossRef]

19. Lee, S.Y.; Kim, Y.K.; Kim, Y.S.; Na, E.J.; Kim, Y.J.; Oem, J.K. Intergenic recombination in feline calicivirus associated with a hemorrhagic-like disease in the Republic of Korea. Acta Virol. 2021, 65, 232-236. [CrossRef]

20. Radford, A.D.; Bennett, M.; McArdle, F.; Dawson, S.; Turner, P.C.; Glenn, M.A.; Gaskell, R.M. The use of sequence analysis of a feline calicivirus $(\mathrm{fcv})$ hypervariable region in the epidemiological investigation of $\mathrm{fcv}$ related disease and vaccine failures. Vaccine 1997, 15, 1451-1458. [CrossRef]

21. Afonso, M.M.; Pinchbeck, G.L.; Smith, S.L.; Daly, J.M.; Gaskell, R.M.; Dawson, S.; Radford, A.D. A multi-national european cross-sectional study of feline calicivirus epidemiology, diversity and vaccine cross-reactivity. Vaccine 2017, 35, 2753-2760. [CrossRef]

22. Smith, S.L.; Afonso, M.M.; Pinchbeck, G.L.; Gaskell, R.M.; Dawson, S.; Radford, A.D. Temporally separated feline calicivirus isolates do not cluster phylogenetically and are similarly neutralised by high-titre vaccine strain $\mathrm{fcv}$-f9 antisera in vitro. J. Feline Med. Surg. 2020, 22, 602-607. [CrossRef] [PubMed]

23. Marsilio, F.; Di Martino, B.; Decaro, N.; Buonavoglia, C. A novel nested pcr for the diagnosis of calicivirus infections in the cat. Vet. Microbiol. 2005, 105, 1-7. [CrossRef] [PubMed]

24. Ramakrishnan, M.A. Determination of 50\% endpoint titer using a simple formula. World J. Virol. 2016, 5, 85-86. [CrossRef] [PubMed]

25. Coyne, K.P.; Jones, B.R.; Kipar, A.; Chantrey, J.; Porter, C.J.; Barber, P.J.; Dawson, S.; Gaskell, R.M.; Radford, A.D. Lethal outbreak of disease associated with feline calicivirus infection in cats. Vet. Rec. 2006, 158, 544-550. [CrossRef] [PubMed]

26. Wardley, R.C.; Gaskell, R.M.; Povey, R.C. Feline respiratory viruses-Their prevalence in clinically healthy cats. J. Small Anim. Pract. 1974, 15, 579-586. [CrossRef] 
27. Coutts, A.J.; Dawson, S.; Willoughby, K.; Gaskell, R.M. Isolation of feline respiratory viruses from clinically healthy cats at UK cat shows. Vet. Rec. 1994, 135, 555-556.

28. Helps, C.R.; Lait, P.; Damhuis, A.; Bjornehammar, U.; Bolta, D.; Brovida, C.; Chabanne, L.; Egberink, H.; Ferrand, G.; Fontbonne, A.; et al. Factors associated with upper respiratory tract disease caused by feline herpesvirus, feline calicivirus, chlamydophila felis and bordetella bronchiseptica in cats: Experience from 218 european catteries. Vet. Rec. 2005, 156, 669-673. [CrossRef] [PubMed]

29. Dall'Ara, P.; Labriola, C.; Sala, E.; Spada, E.; Magistrelli, S.; Lauzi, S. Prevalence of serum antibody titres against feline panleukopenia, herpesvirus and calicivirus infections in stray cats of milan, italy. Prev. Vet. Med. 2019, 167, 32-38. [CrossRef]

30. Wang, K.; Pei, Z.; Hu, G. First report of feline calicivirus (fcv) infection in stray cats in northeast china. Pol. J. Vet. Sci. 2017, 20, 595-598. [CrossRef]

31. Liu, C.; Liu, Y.; Liu, D.; Guo, D.; Liu, M.; Li, Y.; Qu, L. Complete genome sequence of feline calicivirus strain hrb-ss from a cat in heilongjiang province, northeastern China. Genome Announc. 2014, 2, e00698-14. [CrossRef] [PubMed]

32. Wang, Z.; Xin, T.; Wei, J.; Jiang, Y.; Liu, X.; Song, W.; Guo, X.; Yuan, W.; Cui, Y.; Zhu, H.; et al. Isolation and phylogenetic analysis of strains of feline calicivirus in beijing, China. Arch. Virol. 2021, 166, 2521-2527. [CrossRef] [PubMed]

33. Seal, B.S.; Neill, J.D. Capsid protein gene sequence of feline calicivirus isolates 255 and llk: Further evidence for capsid protein configuration among feline caliciviruses. Virus Genes 1995, 9, 183-187. [CrossRef] [PubMed]

34. Mathijs, E.; Muylkens, B.; Mauroy, A.; Ziant, D.; Delwiche, T.; Thiry, E. Experimental evidence of recombination in murine noroviruses. J. Gen. Virol. 2010, 91, 2723-2733. [CrossRef] [PubMed]

35. Ludwig-Begall, L.F.; Mauroy, A.; Thiry, E. Norovirus recombinants: Recurrent in the field, recalcitrant in the lab-A scoping review of recombination and recombinant types of noroviruses. J. Gen. Virol. 2018, 99, 970-988. [CrossRef]

36. Coyne, K.P.; Reed, F.C.; Porter, C.J.; Dawson, S.; Gaskell, R.M.; Radford, A.D. Recombination of feline calicivirus within an endemically infected cat colony. J. Gen. Virol. 2006, 87, 921-926. [CrossRef] [PubMed] 